\title{
Excessive anterior tibial translation in the contralateral uninjured limb is significantly associated with ramp lesion in anterior cruciate ligament injury
}

Kazuki Asai, Junsuke Nakase*, Rikuto Yoshimizu, Mitsuhiro Kimura and Hiroyuki Tsuchiya

\begin{abstract}
Purpose: This study aimed to evaluate the excessive anterior tibial translation (ATT) and muscle strength of patients with ramp lesions. We hypothesised that the higher ATT, lower hamstring-to-quadriceps (HQ) ratio, and higher flexion peak torque influenced by semimembranosus would be associated with ramp lesions.

Methods: One hundred and twenty-one patients who underwent anterior cruciate ligament (ACL) reconstruction were retrospectively evaluated. Clinical evaluation included ATT of the contralateral uninjured limb measured using a KT-1000 arthrometer, the knee flexor and extensor muscle strength of the contralateral uninjured limb at $60 \% \mathrm{~s}$ and $180 \%$ s of an angular velocity measured using an isokinetic dynamometer, and $\mathrm{HQ}$ ratio at $60 \% \mathrm{~s}$ and $180 \% \mathrm{~s}$ during the preoperative state. Binary stepwise logistic regression analysis was performed to evaluate the risk factors of ramp lesions.

Results: Ramp lesions were found in 27 cases of ACL injuries (27/121, 22.3\%). Male sex (odds ratio [OR], 2.913; 95\% confidence interval $[\mathrm{Cl}], 1.090-7.787 ; \mathrm{P}=0.033)$, longer time between injury to surgery $(\mathrm{OR}, 2.225 ; 95 \% \mathrm{Cl}, 1.074-$ 4.608; $P=0.031)$, and higher ATT in the contralateral uninjured $\operatorname{limb}(O R, 1.502 ; 95 \% \mathrm{Cl}, 1.046-2.159 ; \mathrm{P}=0.028)$ were indicated as the independent risk factors of the presence of ramp lesion associated with an $A C L$ injury.
\end{abstract}

Conclusions: Male sex, longer period from injury to surgery, and higher ATT in the contralateral uninjured limb were significantly associated with ramp lesion. These findings are advantageous for identifying patients with a greater risk of developing a ramp lesion with an $\mathrm{ACL}$ injury in the clinical setting.

Level of evidence: Level IV

Keywords: Anterior cruciate ligament injury, Ramp lesion, Meniscus injury, Excessive anterior tibial translation, Muscle strength

\section{Background}

Ramp lesion is a specific type of meniscus lesion such as a meniscocapsular tear of the posterior horn of the medial meniscus (PHMM) associated with anterior cruciate ligament (ACL) injury, which is called a "hidden lesion" [18].

${ }^{*}$ Correspondence: nakase1007@yahoo.co.jp

Department of Orthopaedic Surgery, Graduate School of Medical Science, Kanazawa University, 13-1 Takara-machi, Kanazawa 920-8641, Japan
Ramp lesions accounted for $9 \%-24 \%$ of all ACL injuries $[2,10,19]$. Some studies have reported that longer time from injury to surgery, young age, and contact injury mechanism were risk factors for ramp lesions $[1,10,19]$. In addition, George et al. reported that the presence of bone marrow oedema of the posteromedial tibia or a lateral meniscal tear was associated with the presence of ramp lesions [1]. However, the mechanism and risk factors of ramp lesions remain unclear. 
While the precise mechanisms through which these lesions manifest are not entirely understood, some studies have hypothesised that excessive anterior tibial translation (ATT) and semimembranosus contraction in response to ACL injury possibly cause a ramp lesion [4, 7, 17, 21]. Biomechanical studies have shown that ATT increases following ACL injury, allowing the PHMM to be trapped between the femoral and tibial condyles, acting as a wedge against the tibia, thereby significantly increasing forces in the PHMM $[9,11,13]$. The quadriceps and hamstrings apply antagonist forces to the tibia and play an important role in knee stabilisation [16]. Therefore, imbalance of the quadriceps and hamstring muscles strength potentially causes excessive ATT [6].

To the best of our knowledge, only a few studies have focused on knee laxity and muscle strength in patients with ramp lesions. We aimed to evaluate the characteristics of patients with ramp lesions with a special focus on excessive ATT and hamstring and quadriceps strength. Since it was difficult to assess muscle strength precisely in ACL-injured knees during the preoperative state due to pain and fear, we assessed muscle strength of the contralateral uninjured limb in this study. We hypothesised that the higher ATT, lower hamstring-to-quadriceps (HQ) ratio, and higher flexion peak torque influenced by semimembranosus would be found in patients with ramp lesions associated with an ACL injury.

\section{Methods}

\section{Study design and patients}

One hundred sixty-one patients who underwent ACL reconstruction at our hospital between January 2017 and December 2020 were retrospectively evaluated. ACL injury was diagnosed using magnetic resonance imaging (MRI) and clinical assessments such as the Lachman test and pivot shift test. In all patients, the quadriceps and hamstrings muscle strengths were assessed on the day before surgery. ATT of the uninjured contralateral knee was also assessed as it was difficult to infer the true ATT of the ACL-injured knee during the preinjury state. A previous study found $<3 \%$ difference in peak torque values between dominant and nondominant limbs, which is the same as the accuracy of the force measuring system of isokinetic torques of 3\% [5]. Therefore, we considered that it might be helpful to measure the muscle strength of the contralateral uninjured limb to estimate the strength of the injured limb. The exclusion criteria were ACL reinjury, multi-ligament injuries requiring other ligament surgeries, and history of contralateral ACL injury or other knee surgery. The cases in which muscle strength assessment could not be performed before surgery were also excluded. After excluding 40 patients, 121 cases were finally analysed (age, $23.5 \pm 11.6$ years; body mass index
[BMI], $23.0 \pm 3.3 \mathrm{~kg} / \mathrm{m}^{2}$; and time from injury to surgery, $80 \pm 93$ days). The clinical assessments were compared between the ramp and non-ramp lesion groups. This study was approved by the ethics committee of our hospital (2745-2), and informed consent was obtained from each patient.

\section{Surgical technique}

All surgeries were performed by a single senior surgeon. The medial and lateral meniscal lesions were evaluated before ACL reconstruction using $30^{\circ}$ arthroscopes via an anterolateral portal with probing. The medial meniscus was assessed at $90^{\circ}$ of flexion, and the lateral meniscus (LM) was assessed in the figure-of-4 position. After systematic arthroscopic evaluation for meniscal lesions, the presence of ramp lesions was assessed [20]. First, the meniscocapsular junction was directly assessed by intercondylar viewing with $30^{\circ}$ arthroscope; in some cases, probing was performed through the posteromedial portal. The arthroscope was passed through the anterolateral portal and inserted between the posterior cruciate ligament and the medial wall of the intercondylar notch into the posteromedial compartment with the knee in $90^{\circ}$ flexion and slightly externally rotated (Fig. 1A). Inspection through the posteromedial portal may detect more ramp lesions [18], but this requires more time and is significantly invasive for a screening procedure. Therefore, this technique was used in only suspected cases as needed. Second, the medial meniscus was evaluated through the anterolateral portal with probing (Fig. 1B).

\section{Clinical assessment}

Clinical evaluation included ATT of the contralateral uninjured limb, the knee flexor and extensor muscle strength at $60 \% \mathrm{~s}$ and $180 \% \mathrm{~s}$ of angular velocity, and the HQ ratio at $60 \%$ s and $180 \%$ during the preoperative state. ATT was measured by a KT-1000 arthrometer (MEDmetric Corp., San Diego, CA, USA) with manual max by the orthopaedic surgeon who was blinded to any other information, such as MRI findings. A physiotherapist who was also blinded to any other findings used an isokinetic dynamometer (BIODEX System 4; BIODEX Company, Shirley, NY, USA) to assess flexion and extension peak torque at $60^{\circ} / \mathrm{s}$ and $180^{\circ} / \mathrm{s}$. After warming up using an ergometer for $5 \mathrm{~min}$ and practicing two sets of flexion and extension exercises thrice, the patients participated in the measurement test. The patients were allowed sufficient rest time between the sets. The measurement range was $0^{\circ}-100^{\circ}$ for the knee joint, and the lower leg was gravity-corrected. The average knee flexion and extension peak torque were calculated by three flexion and extension exercises at the production set. The HQ ratio was defined as hamstring muscle strength 


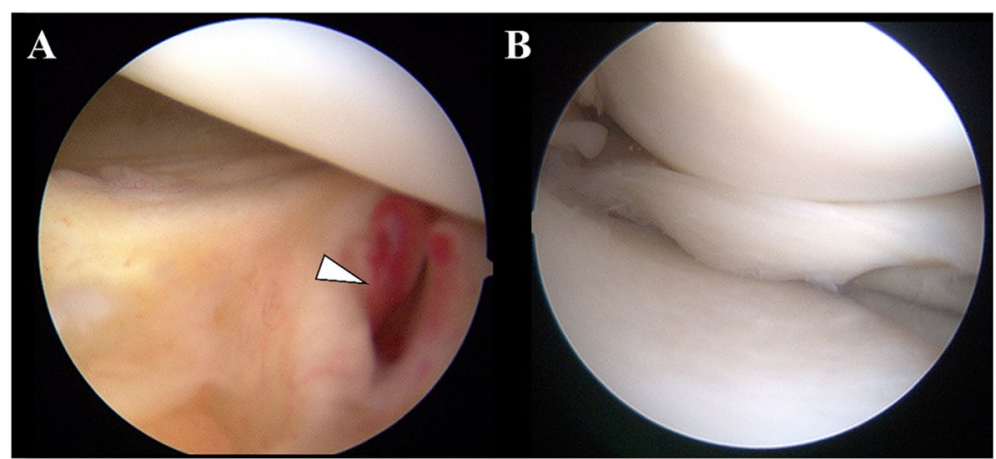

Fig. 1 Systematic arthroscopic evaluation of ramp lesions. A The meniscocapsular junction is directly assessed by intercondylar viewing. B Second, the medial meniscus is evaluated through the anterolateral portal with probing. Arrowhead marks meniscocapsular tear of the posterior horn of the medial meniscus

(flexion peak torque)/quadriceps muscle strength (extension peak torque).

\section{Statistical analysis}

Continuous variables were presented as mean \pm standard deviation. According to the results of Shapiro-Wilk test used to test the normality of their distribution, age, BMI, ATT of the uninjured contralateral limb, and the flexion peak torque at $180^{\circ} / \mathrm{s}$ were compared using Mann-Whitney $U$ test between the ramp and nonramp lesion groups. Chi-squared test was used to compare sex, time from injury to ACL reconstruction $(<3$, $3-6$, or $>6$ months), LM tear, and mechanism of injury (contact or non-contact) between the groups. The flexion peak torque at $60 \%$, extension peak torque at $60 \%$ and $180^{\circ} \% \mathrm{~s}$, and the HQ ratio at $60^{\circ} \% \mathrm{~s}$ and $180^{\circ} / \mathrm{s}$ were compared using independent $t$ test. Binary stepwise logistic regression analysis was performed to evaluate the risk factors of the presence of ramp lesions. The predictive factors were as follows: age at surgery, sex, BMI, time from injury to ACL reconstruction $(<3,3-6$, or $>6$ months), LM tear, mechanism of injury (contact or non-contact), ATT in the uninjured contralateral limb, the knee flexion and extension peak torque at $60 \% \mathrm{~s}$ and $180 \% \mathrm{~s}$, and the HQ ratio at $60 \% \mathrm{~s}$ and $180 \%$ s during the preoperative period. The significance level was set at $\mathrm{p}<0.05$, and the $95 \%$ confidence interval $(\mathrm{CI})$ was calculated. All statistical analyses were performed using IBM SPSS Statistics for Windows, ver. 24 software (IBM Corp., Armonk, NY, USA).

Post-hoc power analysis was performed with a chisquared goodness-of-fit test with an $\alpha$ error of 0.05 and a $\beta$ error of 0.2 to detect the differences in the incidence of significant risk factors between the groups. Based on these calculations, the power of significant factors in this study was $>0.9$. To detect differences in mean values of significant risk factors between the groups, post-hoc power analysis was also performed with an $\alpha$ error of 0.05 and a $\beta$ error of 0.2 . The power of the mean values of significant risk factors was $>0.8$. These post-hoc power analyses were performed using $G^{*}$ power 3.1 software (Heinrich-Heine University Dusseldorf, Dusseldorf, Germany).

\section{Results}

In this study, $70.2 \%(85 / 121)$ of ACL injury cases had a meniscal tear (Fig. 2). Ramp lesions were found in 27 cases of ACL injury (27/121, 22.3\%). Among the cases of ramp lesion, $44.4 \%$ (12/27 cases) had a concomitant LM tear.

\section{Demographics of patients with a ramp lesion}

The characteristics of patients with and without a ramp lesion are presented in Table 1. The ramp lesion group showed significantly higher predilection in men, longer time from injury to surgery, and higher ATT in the contralateral uninjured limb than the non-ramp lesion group $(\mathrm{P}=0.044,0.001$, and 0.034 , respectively).

\section{Risk factors of ramp lesions}

On logistic regression analysis, male sex (odds ratio [OR], 2.913; 95\% CI, 1.090-7.787; $\mathrm{P}=0.033)$, longer time from injury to surgery $(\mathrm{OR}, 2.225 ; 95 \% \mathrm{CI}$, 1.074-4.608; $\mathrm{P}=0.031$ ), and higher ATT in the contralateral uninjured limb (OR, 1.502; 95\% CI, 1.0462.159; $\mathrm{P}=0.028$ ) were indicated as independent risk factors for the presence of ramp lesions associated with ACL injury (Table 2). 


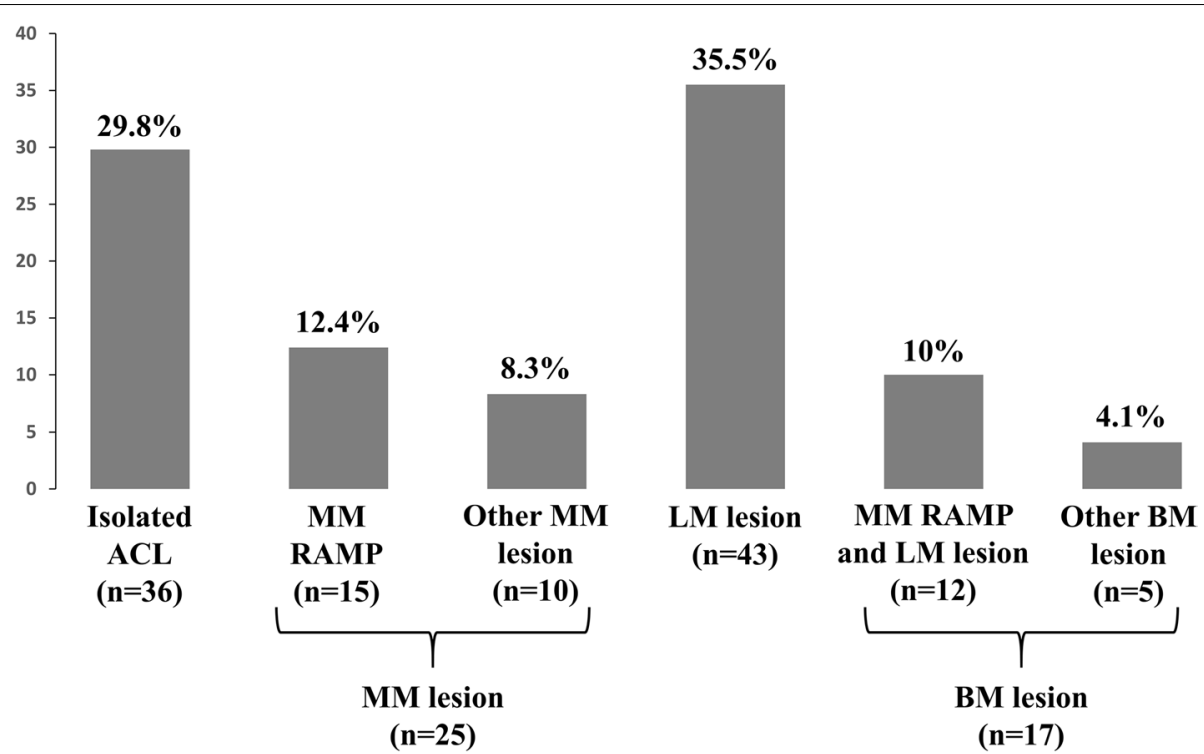

Fig. 2 Prevalence of concomitant meniscal lesion with an ACL injury. Meniscus injuries and specific types of lesions, including ramp lesions, are presented. MM, medial meniscus; LM, lateral meniscus; BM, bimeniscal; $A C L$, anterior cruciate ligament

\section{Discussion}

On logistic regression analysis, male sex, long time from injury to surgery, and higher ATT in the contralateral uninjured limb were significantly associated with ramp lesions. Although some of our hypotheses were confirmed, others about muscle strength were not.

This study also found excessive ATT in the contralateral uninjured limb as an indicator of knee condition during the pre-ACL injury state and as a risk factor of ramp lesion. Our findings were supported by those of previous studies that advocated a similar mechanism for developing a ramp lesion: excessive ATT and semimembranosus contraction during ACL injury may lead to excessive loading of the PHMM, meniscotibial ligament, and meniscocapsular junction, resulting in a ramp lesion $[4,7,17]$. Saygi et al. found that semimembranosus reflexively contracts in response to PHMM translation during ATT and is responsible for posterior shifting

Table 1 Patient demographics

\begin{tabular}{|c|c|c|c|}
\hline & $\begin{array}{l}\text { Ramp lesion } \\
\text { ( } 27 \text { cases) }\end{array}$ & $\begin{array}{l}\text { Non-ramp lesion } \\
\text { (94 cases) }\end{array}$ & P-value \\
\hline Age (y) & $21.9 \pm 8.0$ & $24.0 \pm 12.4$ & n.s \\
\hline Sex (Male:Female) & $18: 9$ & $43: 51$ & 0.044 \\
\hline $\mathrm{BMI}\left(\mathrm{kg} / \mathrm{m}^{2}\right)$ & $22.4 \pm 2.1$ & $23.0 \pm 3.4$ & n.s \\
\hline \multirow[t]{3}{*}{ Time from injury to surgery (months) } & $0-3: 14$ & $0-3: 79$ & \multirow[t]{3}{*}{0.001} \\
\hline & $3-6: 11$ & $3-6: 10$ & \\
\hline & $>6: 2$ & $>6: 5$ & \\
\hline Lateral meniscus tear & $12(44.4 \%)$ & $47(50.0 \%)$ & n.s \\
\hline $\begin{array}{l}\text { Mechanism of injury } \\
\text { (Contact:Non-contact) }\end{array}$ & $5: 22$ & $30: 64$ & n.s \\
\hline Anterior tibial translation (mm) & $5.4 \pm 1.6$ & $4.7 \pm 1.1$ & 0.034 \\
\hline Flexion peak torque $\left(60^{\circ} / \mathrm{s}\right)$ & $59.5 \pm 21.8$ & $59.6 \pm 21.2$ & n.s \\
\hline Extension peak torque $\left(60^{\circ} / \mathrm{s}\right)$ & $140.3 \pm 40.1$ & $133.8 \pm 42.5$ & n.s \\
\hline $\mathrm{HQ}$ ratio $\left(60^{\circ} / \mathrm{s}\right)$ & $0.42 \pm 0.09$ & $0.45 \pm 0.09$ & n.s \\
\hline Flexion peak torque $\left(180^{\circ} / \mathrm{s}\right)$ & $52.0 \pm 20.4$ & $51.0 \pm 18.8$ & n.s \\
\hline Extension peak torque $\left(60^{\circ} / \mathrm{s}\right)$ & $94.5 \pm 27.7$ & $90.9 \pm 30.5$ & n.s \\
\hline HQ ratio $\left(180^{\circ} / \mathrm{S}\right)$ & $0.54 \pm 0.10$ & $0.56 \pm 0.10$ & n.s \\
\hline
\end{tabular}

$B M I$ body mass index, $H Q$ hamstrings strength-to-quadriceps strength, n.s., not significant 
Table 2 Results of logistic regression analysis of ramp lesions

\begin{tabular}{llllll}
\hline & Odds ratio & $\boldsymbol{\beta}$ & SE & $\mathbf{9 5 \%} \mathbf{C l}$ & P-value \\
\hline Sex (male) & 2.913 & 1.069 & 0.502 & $1.090-7.787$ & $\mathbf{0 . 0 3 3}$ \\
$\begin{array}{l}\text { Time from injury to } \\
\text { surgery }\end{array}$ & 2.225 & 0.800 & 0.371 & $1.074-4.608$ & $\mathbf{0 . 0 3 1}$ \\
$\begin{array}{c}\text { Anterior tibial trans- } \\
\text { lation }\end{array}$ & 1.502 & 0.407 & 0.185 & $1.046-2.159$ & $\mathbf{0 . 0 2 8}$ \\
HQ ratio & 0.006 & -5.190 & 2.688 & $0.000-1.081$ & n.s \\
\hline
\end{tabular}

Likelihood ratio test, $\mathrm{P}=0.001$

Nagelkerke $\mathrm{R}^{2}=0.225$

$S E$ standard error, $\mathrm{Cl}$ confidence interval, $\mathrm{HQ}$ hamstrings strength-to-quadriceps strength, n.s., not significant

of the PHMM $[7,15]$. Therefore, we considered that the patients who presented with excessive ATT in the contralateral uninjured limb carry a risk for ramp lesions even if the preoperative side-to-side laxity is not high. The incidence of ramp lesions in our study was similar to that in a previous large cohort study by Sonnery et al., who reported ramp lesions in $23.9 \%(769 / 3214$ cases) of ACL injury cases [19]. They revealed that male sex and longer chronic period between injury to surgery were risk factors of ramp lesions [19]. However, a previous study also found that young age, contact injury, and concomitant LM injury were risk factors of ramp lesions, whereas these factors were not significant in the present study $[1,19]$.

We also explored the semimembranosus contraction as one of the causes of ramp lesions. Recently, many studies have investigated the association between the semimembranosus muscle and ramp lesions [3, 4, 21]. Based on histological findings, DePhillipo et al. reported that the branch from the anterior arm of the semimembranosus muscle tendon is attached to the posterior inferior margin of the medial meniscus [4]. Vieira et al. performed an arthroscopic assessment and found that applying load to the semimembranosus tendon leads to posterior translation of the PHMM and stretching of the meniscocapsular region [21]. Cavaignac et al. noted that the capsular arm of the semimembranosus tendon protruded over the joint capsule, and adipose tissue was located in between the capsular branch of the semimembranosus and the medial meniscus, including the common attachment of the meniscocapsular and meniscotibial ligaments [3]. However, contrary to our hypothesis, there was no significant difference in the peak torque of knee flexion, which may be influenced by the semimembranosus muscle. The capsular branch of the semimembranosus was subjected to the milder mechanical stress than the direct arm of the semimembranosus inserted into the tibia because of their secondary mechanical role [3]. Therefore, our evaluation methods of merely assessing the hamstrings muscle strength might have been inadequate to detect the difference in activity of the capsular branch of the semimembranosus between the knees with and without ramp lesions.

The findings of the present study should be interpreted with consideration of few limitations. First, this study had possible selection bias due to the exclusion of patients in whom muscle strength was not assessed. Second, there was a relatively small number of cases in this study. Third, we evaluated the muscle strength of contralateral uninjured limbs as an indicator of the knee condition during the pre-ACL injury state. Previous studies found less than $3 \%$ differences in the peak torque value and $1 \%$ differences in the HQ ratio between dominant and nondominant legs $[5,12,14]$. Lee et al. demonstrated a $50 \%$ reduction of thigh muscle strength in a group of patients with chronic ACL injury. However, they still observed HQ ratios comparable to those of a healthy control group [8]. Therefore, we considered that evaluating the HQ ratio of the contralateral uninjured limb was rather reasonable for assessing the muscle strength of knee extension and flexion before ACL reconstruction. Fourth, this study did not consider the influence of time from injury to surgery. A previous study reported decreased muscle strength in chronic ACL-injured knees [8]. Despite these limitations, the results of this study suggested that the patients with a predisposition to excessive ATT due to knee laxity or a lower $\mathrm{HQ}$ ratio carry the risk of developing a ramp lesion with an ACL injury. Moreover, a long time from injury to surgery was a risk factor of ramp lesions. These findings might help surgeons counsel patients in the setting of ACL tears with concomitant ramp lesions.

\section{Conclusions}

Male sex, a longer period from injury to surgery, and higher ATT of the contralateral uninjured limb were significantly associated with ramp lesions. These findings are advantageous for identifying patients with a greater risk of developing a ramp lesion with an ACL injury in the clinical setting.

\section{Abbreviations}

ACL: Anterior cruciate ligament; ATT: Anterior tibial translation; BMI: Body mass index; Cl: Confidence interval; HQ: Hamstring-to-quadriceps; LM: Lateral meniscus; MRI: Magnetic resonance imaging; OR: Odds ratio; PHMM: Posterior horn of the medial meniscus.

\section{Authors' contributions}

All authors contributed to the study conception and design. Material preparation and data collection and analysis were performed by K. A., J. N., R. Y., M. K., and T. S. The first draft of the manuscript was written by K. A., and all authors commented on previous versions of the manuscript. All authors read and approved the final manuscript. 


\section{Funding}

The authors do not have any financial disclosures.

\section{Availability of data and materials}

The datasets used and analysed during the current study are available from the corresponding author on reasonable request.

\section{Declarations}

Ethics approval and consent to participate

This study was approved by the ethics committee of our hospital. Informed consent was obtained from each patient.

\section{Consent for publication}

Not applicable.

\section{Competing interests}

The authors declare no conflicts of interest.

Received: 19 May 2021 Accepted: 16 July 2021

Published online: 23 July 2021

\section{References}

1. Balazs GC, Greditzer HGt, Wang D, Marom N, Potter HG, Marx RG, et al (2019) Ramp Lesions of the Medial Meniscus in Patients Undergoing Primary and Revision ACL Reconstruction: Prevalence and Risk Factors. Orthop J Sports Med 7:2325967119843509

2. Bollen SR (2010) Posteromedial meniscocapsular injury associated with rupture of the anterior cruciate ligament: a previously unrecognised association. J Bone Joint Surg Br 92:222-223

3. Cavaignac E, Sylvie R, Teulières M, Fernandez A, Frosch KH, GomezBrouchet A et al (2021) What Is the Relationship Between the Distal Semimembranosus Tendon and the Medial Meniscus? A Gross and Microscopic Analysis From the SANTI Study Group. Am J Sports Med 49:459-466

4. DePhillipo NN, Moatshe G, Chahla J, Aman ZS, Storaci HW, Morris ER et al (2019) Quantitative and Qualitative Assessment of the Posterior Medial Meniscus Anatomy: Defining Meniscal Ramp Lesions. Am J Sports Med 47:372-378

5. Farrell M, Richards JG (1986) Analysis of the reliability and validity of the kinetic communicator exercise device. Med Sci Sports Exerc 18:44-49

6. Hiemstra LA, Webber S, MacDonald PB, Kriellaars DJ (2004) Hamstring and quadriceps strength balance in normal and hamstring anterior cruciate ligament-reconstructed subjects. Clin J Sport Med 14:274-280

7. Kaplan EB (1962) Some aspects of functional anatomy of the human knee joint. Clin Orthop 23:18-29

8. Lee DH, Lee JH, Jeong HJ, Lee SJ (2015) Lack of Correlation between Dynamic Balance and Hamstring-to-Quadriceps Ratio in Patients with Chronic Anterior Cruciate Ligament Tears. Knee Surg Relat Res 27:101-107
9. Lee JJ, Choi YJ, Shin KY, Choi CH (2011) Medial meniscal tears in anterior cruciate ligament-deficient knees: effects of posterior tibial slope on medial meniscal tear. Knee Surg Relat Res 23:227-230

10. Liu X, Feng H, Zhang H, Hong L, Wang XS, Zhang J (2011) Arthroscopic prevalence of ramp lesion in 868 patients with anterior cruciate ligament injury. Am J Sports Med 39:832-837

11. Łuczkiewicz P, Daszkiewicz K, Witkowski W, Chróścielewski J, Zarzycki W (2015) Influence of meniscus shape in the cross sectional plane on the knee contact mechanics. J Biomech 48:1356-1363

12. Lund-Hanssen H, Gannon J, Engebretsen L, Holen K, Hammer S (1996) Isokinetic muscle performance in healthy female handball players and players with a unilateral anterior cruciate ligament reconstruction. Scand J Med Sci Sports 6:172-175

13. Papageorgiou CD, Gil JE, Kanamori A, Fenwick JA, Woo SL, Fu FH (2001) The biomechanical interdependence between the anterior cruciate ligament replacement graft and the medial meniscus. Am J Sports Med 29:226-231

14. Risberg MA, Steffen K, Nilstad A, Myklebust G, Kristianslund E, Moltubakk MM et al (2018) Normative Quadriceps and Hamstring Muscle Strength Values for Female, Healthy, Elite Handball and Football Players. J Strength Cond Res 32:2314-2323

15. Saygi B, Yildirim Y, Berker N, Ofluoglu D, Karadag-Saygi E, Karahan M (2005) Evaluation of the neurosensory function of the medial meniscus in humans. Arthroscopy 21:1468-1472

16. Shelburne KB, Torry MR, Pandy MG (2005) Effect of muscle compensation on knee instability during ACL-deficient gait. Med Sci Sports Exerc 37:642-648

17. Sims WF, Jacobson KE (2004) The posteromedial corner of the knee: medial-sided injury patterns revisited. Am J Sports Med 32:337-345

18. Sonnery-Cottet B, Conteduca J, Thaunat M, Gunepin FX, Seil R (2014) Hidden lesions of the posterior horn of the medial meniscus: a systematic arthroscopic exploration of the concealed portion of the knee. Am J Sports Med 42:921-926

19. Sonnery-Cottet B, Praz C, Rosenstiel N, Blakeney WG, Ouanezar H, Kandhari V et al (2018) Epidemiological Evaluation of Meniscal Ramp Lesions in 3214 Anterior Cruciate Ligament-Injured Knees From the SANTI Study Group Database: A Risk Factor Analysis and Study of Secondary Meniscectomy Rates Following 769 Ramp Repairs. Am J Sports Med 46:3189-3197

20. Tashiro Y, Mori T, Kawano T, Oniduka T, Arner JW, Fu FH et al (2020) Meniscal ramp lesions should be considered in anterior cruciate ligamentinjured knees, especially with larger instability or longer delay before surgery. Knee Surg Sports Traumatol Arthrosc 28:3569-3575

21. Vieira TD, Pioger C, Frank F, Saithna A, Cavaignac E, Thaunat M et al (2019) Arthroscopic Dissection of the Distal Semimembranosus Tendon: An Anatomical Perspective on Posteromedial Instability and Ramp Lesions. Arthrosc Tech 8:e987-e991

\section{Publisher's Note}

Springer Nature remains neutral with regard to jurisdictional claims in published maps and institutional affiliations.

\section{Submit your manuscript to a SpringerOpen ${ }^{\odot}$ journal and benefit from:}

- Convenient online submission

- Rigorous peer review

- Open access: articles freely available online

- High visibility within the field

- Retaining the copyright to your article

Submit your next manuscript at springeropen.com 